\title{
Longer Tendon-Bone Distances of the A2 and A4 Annular Pulleys in Experienced High-Level Sport Climbers: Injury or Adaptation?
}

Xeber Iruretagoiena, $\mathrm{PT}, \mathrm{PhD}^{1,2,3}$; Javier De la Fuente, $\mathrm{MD}, \mathrm{PhD}^{4}$; Elena Sonsoles Rodríguez-López, PT, DO, $\mathrm{PhD}^{1}$; Fernando Davila, $\mathrm{MD}^{4}$; Asier Dorronsoro, $\mathrm{PT}, \mathrm{MSc}^{2}$; Leire Goenaga, $\mathrm{PT}^{2}$; Marc Blasi, PT, MD, $\mathrm{MSc}^{5}$

${ }^{1}$ Department of Physical Therapy, Universidad Camilo José Cela, Madrid, Spain; ${ }^{2}$ Eskura Osasun Zentroa, Beasain, Spain; ${ }^{3}$ Department of Physical Therapy, Universidad of Deusto, San Sebastian, Spain; ${ }^{4}$ Orthopedics Department, Clínica Pakea-Mutualia, San Sebastián, Spain; ${ }^{5}$ Plastic Surgery Department, Hospital Universitari Germans Trias i Pujol, Badalona (Barcelona), Spain

\begin{abstract}
Introduction-Experienced high-level climbers are subject to a number of bone and soft tissue changes over the years and are also among the most exposed to pulley injuries. One of the main consequences of pulley rupture is the separation of the flexor tendons from the subjacent phalanges, also known as bowstringing. The purpose of this study was to determine whether this population has asymptomatic bowstringing of the A2 and/or A4 pulleys as determined by tendon-bone distance (TBD) values when compared to nonclimbers.

Methods - High-resolution ultrasound TBD measurements in active forced flexion were made for the A2 and A4 pulley of the ring finger bilaterally. Participants were 21 asymptomatic sport climbers who had 21 consecutive years of climbing at a level above 9.66 in the International Mountaineering and Climbing Federation difficulty metric scale. Control subjects were 21 age-matched nonclimbers.

Results-A significantly longer TBD-25\% (0.3 mm) and 35\% $(0.4 \mathrm{~mm})$ for the A2 and A4 pulleys, respectively-was found in the experienced climbers group (experienced climbers group: A2 1.6 \pm 0.5 $\mathrm{mm}$ and A4 1.6 $\pm 0.4 \mathrm{~mm}$; nonclimbers group: A2 $1.2 \pm 0.1 \mathrm{~mm}$ and A4 $1.2 \pm 0.2 \mathrm{~mm}$ ).

Conclusions-Our results suggest that bowstringing of A2 and A4 pulleys occurs in asymptomatic experienced high-level climbers, which could be interpreted as either an adaptive mechanism to workloads endured over years of climbing or a consequence of underdiagnosed pulley ruptures.
\end{abstract}

Keywords: ultrasound, bowstringing, noninjured, asymptomatic

\section{Introduction}

Rock climbing is a sport practiced worldwide and has been recently accepted for the 2021 Olympics. ${ }^{1}$ With growing global interest in this sport, there has been an increase in the number of climbers, together with an improvement in climbing equipment and protection measures. These have led to a proportional reduction in the number of injuries ${ }^{2,3}$ and a change in the most frequent injury types, which have shifted from leg injuries associated with traumatic falls ${ }^{1}$ to overuse injuries

Corresponding author: Elena S. Rodríguez, PT, DO, PhD, Universidad Camilo José Cela, Department of Physical Therapy, Madrid, Spain 28692; e-mail: esrodriguez@ucjc.edu.

Submitted for publication October 2020.

Accepted for publication July 2021. mainly affecting the shoulder and fingers. ${ }^{4}$ The most recent reports show that $33 \%$ of all climbing injuries affect the flexor tendon sheath of the hand, especially the A2 and A4 annular finger pulleys of the middle and ring fingers. ${ }^{1,5}$ In adult rock climbers, the latter continues to be the most common. ${ }^{6}$

Another important change in the practice of rock climbing has occurred in relation to sport climbing routes, as historic levels of difficulty have been surpassed and levels continue to rise. ${ }^{7}$ Currently, the hardest sport climbing grade is $9 \mathrm{c}$ measured using the French system, $5.15 \mathrm{c} / \mathrm{d}$ in Yosemite Decimal System, or 12.33 in the metric scale of the International Mountaineering and Climbing Federation (UIAA), ${ }^{8}$ and the increase in difficulty is attributable mainly to developments in strategies and training. Different grading systems exist to rate 
climbing route difficulty, and the UIAA metric scale is a recognized sport-specific scoring system widely used for injury-reporting scientific analysis. ${ }^{8}$

Climbers start training at younger ages, and the trend is that elite climbers are much younger and have more demands and greater workloads, especially at the level of the fingers. ${ }^{9,10}$ At this point, it is important to mention that injury type distribution is different in adolescent compared to adult climbers. ${ }^{11}$ Whereas A2 and A4 pulley ruptures are the most frequent injuries in adult climbers, epiphyseal growth plate fractures to the base of the middle phalanx are the most prevalent injuries in youth climbers. ${ }^{12}$

High workloads on fingers sustained over years lead to an adaptive process in several anatomic components of the fingers. ${ }^{7}$ In adult climbers, adaptive changes in the bone and soft tissues of the fingers have been described. Compared to nonclimbers, radiographic findings of the middle phalanx include $25 \%$ higher cortical proportions, up to $6 \%$ greater cortical widths, and up to $20 \%$ narrower medullary canals. ${ }^{13}$ Overall, the A2 pulley is thicker in $69 \%$ of climbers, whereas the A4 pulley is thicker in $75 \%$ of climbers. The volar plate of the distal interphalangeal joint is also significantly thicker in climbers compared to nonclimbers. ${ }^{7}$ Controversy still exists regarding the thickening of the finger flexor tendons at the proximal phalanx as an adaptive response to workload. ${ }^{7,14}$

High-resolution ultrasound (US) is one of the most suitable diagnostic methods for traumatic finger injuries. ${ }^{15}$ Both qualitative and quantitative US signs have been proposed for the diagnosis of ruptured annular pulleys, tendon-bone distance (TBD) measurement being among the most recommended. ${ }^{16}$ The most accepted TBD threshold to diagnose a complete pulley rupture is 2 mm. ${ }^{1}$ However, in rock climbers, TBDs considered pathological are not always accompanied by clinical symptoms or loss of grip strength. ${ }^{17}$ This has prompted the hypothesis that either adaptive changes or subclinical injuries could generate finger pulley slackening, related to a certain degree of bowstringing evidenced sonographically by an increased TBD.

The main objective of the present study was to determine whether the TBD of the A2 and A4 pulleys is greater in asymptomatic experienced high-level climbers compared to age-matched asymptomatic nonclimbers. As secondary objectives, we also aimed to determine whether 1) TBD differences exist between the A2 and A4 pulleys, 2) TBD differences exist between the dominant and nondominant hands, and 3) asymptomatic experienced high-level climbers may have TBDs over $2 \mathrm{~mm}$.

\section{Methods}

We designed a cross-sectional study in which we recruited noninjured experienced high-level sport climbers to undergo a single US assessment. Inclusion criteria were a lack of clinical symptoms and a minimum

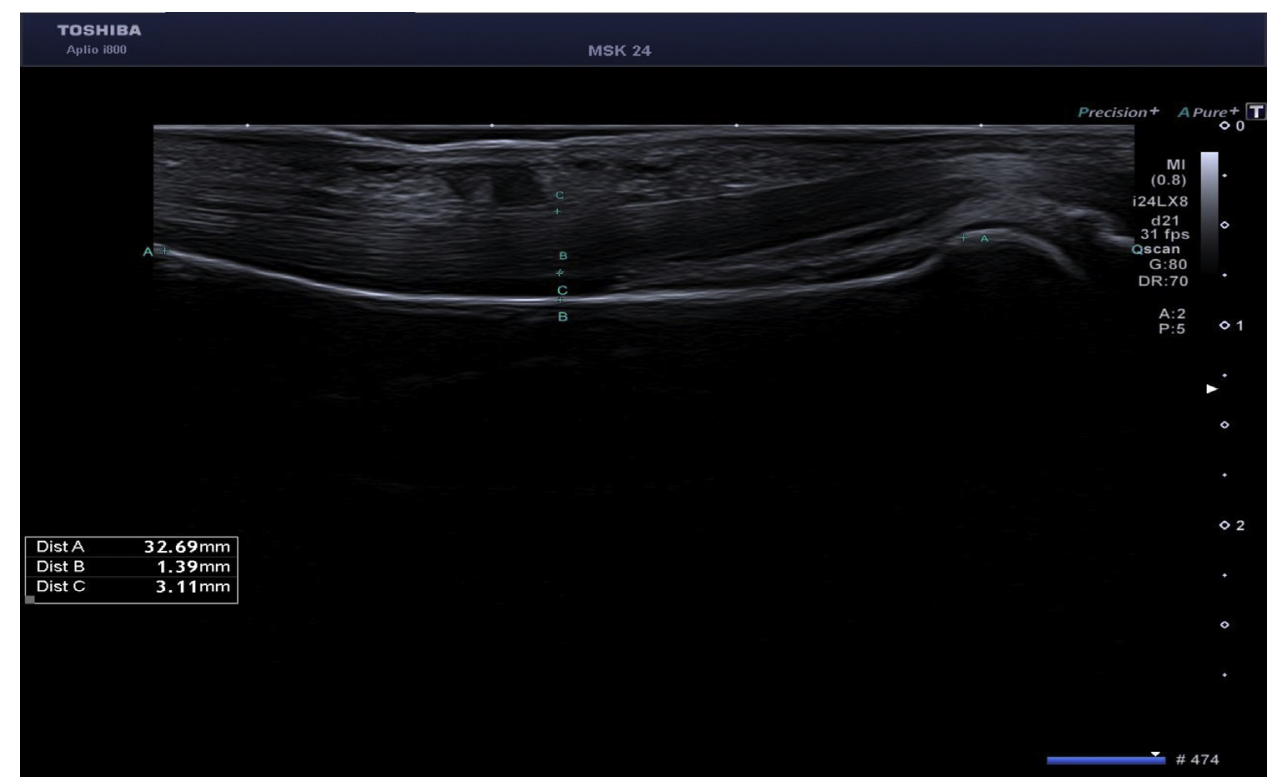

Figure 1. High-resolution ultrasound image of a non-ruptured A2 pulley. The distance B indicates the tendon-bone distance in the midpoint of the proximal phalanx. 
Table 1. Demographic data

\begin{tabular}{|c|c|c|c|c|c|}
\hline & \multicolumn{2}{|c|}{ Climbers $(n=21)$} & \multicolumn{2}{|c|}{ Nonclimbers $(n=21)$} & \multirow[t]{2}{*}{$\mathrm{P}$ value } \\
\hline & $M e a n \pm S D$ & $95 \% C I$ & $M e a n \pm S D$ & $95 \% C I$ & \\
\hline Age (y) & $42 \pm 6$ & $39-45$ & $42 \pm 7$ & $39-45$ & $0.769^{a}$ \\
\hline Weight (kg) & $68 \pm 6$ & $66-71$ & $69 \pm 6$ & $67-72$ & $0.558^{a}$ \\
\hline Height $(\mathrm{cm})$ & $173 \pm 6$ & $171-176$ & $173 \pm 6$ & $171-176$ & $0.914^{a}$ \\
\hline Highest level in sport climbing ${ }^{b}$ & $10.5 \pm 0.3$ & $10.3-10.6$ & - & - & - \\
\hline Years of climbing & $26 \pm 5$ & $23-28$ & - & - & - \\
\hline Years of climbing above 9.66 climbing level & $21 \pm 4$ & $19-23$ & - & - & - \\
\hline
\end{tabular}

${ }^{a} P$ value for comparisons between the 2 study groups \pm Student $\mathrm{t}$ test.

${ }^{b}$ UIAA climbing difficulty metric scale.

of 15 consecutive years of climbing at a difficulty level above grade 9.66 on the UIAA metric scale. ${ }^{8}$ Exclusion criteria were any previous diagnosis of pulley rupture of the ring finger or hand surgery. The study finally included 21 rock climbers. The control group was composed of 21 healthy nonclimbers who also met the exclusion criteria.

The study was conducted in accordance with the ethical standards of the Declaration of Helsinki. ${ }^{18}$ All data were anonymous and confidential. The study protocol received approval by the research ethics committee of the Camilo José Cela University (Spain, EOPPADME, 22 March 2018). All patients were informed in writing of the study's objectives and procedures and provided written informed consent to participate.

General demographic and climbing data were collected from all participants (age, weight, height, highest level in sport climbing, years of climbing, years of climbing above UIAA 9.66 climbing level). All participants underwent a US assessment using a Canon Aplio i800 equipped with a $24 \mathrm{MHz}$ linear transducer (Canon Medical Systems S.A., Madrid, Spain). US image quality is in part determined by transducer frequency, and a minimum of $14 \mathrm{MHz}$ is recommended. ${ }^{19}$ The participant sat in front of the examiner with forearms and hands in supine position and a neutral wrist position. Finger position was standardized as follows: neutral metacarpophalangeal joint, $40^{\circ}$ of flexion of the proximal interphalangeal joint, and $10^{\circ}$ flexion of the distal interphalangeal joint. ${ }^{20} \mathrm{~A}$ counter-resisted force to the distal phalanx was applied while the patient was instructed to execute maximum flexion force of the finger, under the pain threshold. ${ }^{20}$ The TBD of the A2 and A4 pulleys was then measured along the longitudinal axis of the ring finger bilaterally by placing the transducer over the central palmar aspect of the finger, without any tilt and using abundant gel to avoid compression (Figure 1). Measurements were made over the midpoint of the proximal phalanx for the A2 pulley and the medial phalanx for the A4 pulley. ${ }^{21}$

Statistical analysis was performed using SPSS 22.0 software (SPSS Science, Chicago, IL, USA). Descriptive statistics (mean, SD, and 95\% CI for continuous measures) were calculated. The Shapiro-Wilk test confirmed a normal distribution of the quantitative outcome measures $(P>0.05)$. A t-test was used to analyze intergroup comparisons for age, weight, and height. A repeatedmeasure analysis of variance mixed linear effects model was used to compare the TBD measurements of the A2 and A4 pulleys in the fingers of both hands in climber and nonclimber groups (Bonferroni correction was used for post hoc analysis). Percentage differences between climbers and nonclimbers were calculated using the following equation: ([TBD climbers - TBD nonclimbers]/TBD climbers $\times 100$ ). The effect size measure was determined using Cohen's d (large effect, $\mathrm{d}>0.8$; intermediate effect, $\mathrm{d}=0.5-0.8$ ). Bivariate correlations

Table 2. Experienced high-level climber group TBDs above $2 \mathrm{~mm}$ threshold

\begin{tabular}{|c|c|c|c|c|c|c|c|}
\hline \multirow[b]{2}{*}{ Pulley } & \multicolumn{2}{|c|}{$\begin{array}{l}\text { Negative } \\
(<2 \mathrm{~mm})\end{array}$} & \multicolumn{2}{|c|}{$\begin{array}{l}\text { Positive } \\
(>2 \mathrm{~mm})\end{array}$} & \multirow[t]{2}{*}{ Min. TBD (mm) } & \multirow[t]{2}{*}{$\operatorname{Max} . T B D(\mathrm{~mm})$} & \multirow[t]{2}{*}{ Mean $\pm S D$} \\
\hline & $n$ & $\%$ & $n$ & $\%$ & & & \\
\hline $\mathrm{A} 2$ & 34 & 81 & 8 & 19 & 0.9 & 3.3 & $1.5 \pm 0.5$ \\
\hline A4 & 33 & 79 & 9 & 21 & 1 & 3 & $1.6 \pm 0.4$ \\
\hline $\mathrm{A} 2$ and $\mathrm{A} 4$ & 67 & 80 & 17 & 20 & 1 & 3 & $1.6 \pm 0.4$ \\
\hline
\end{tabular}

Min, minimum value; Max, maximum value; TBD, tendon-bone distance. 


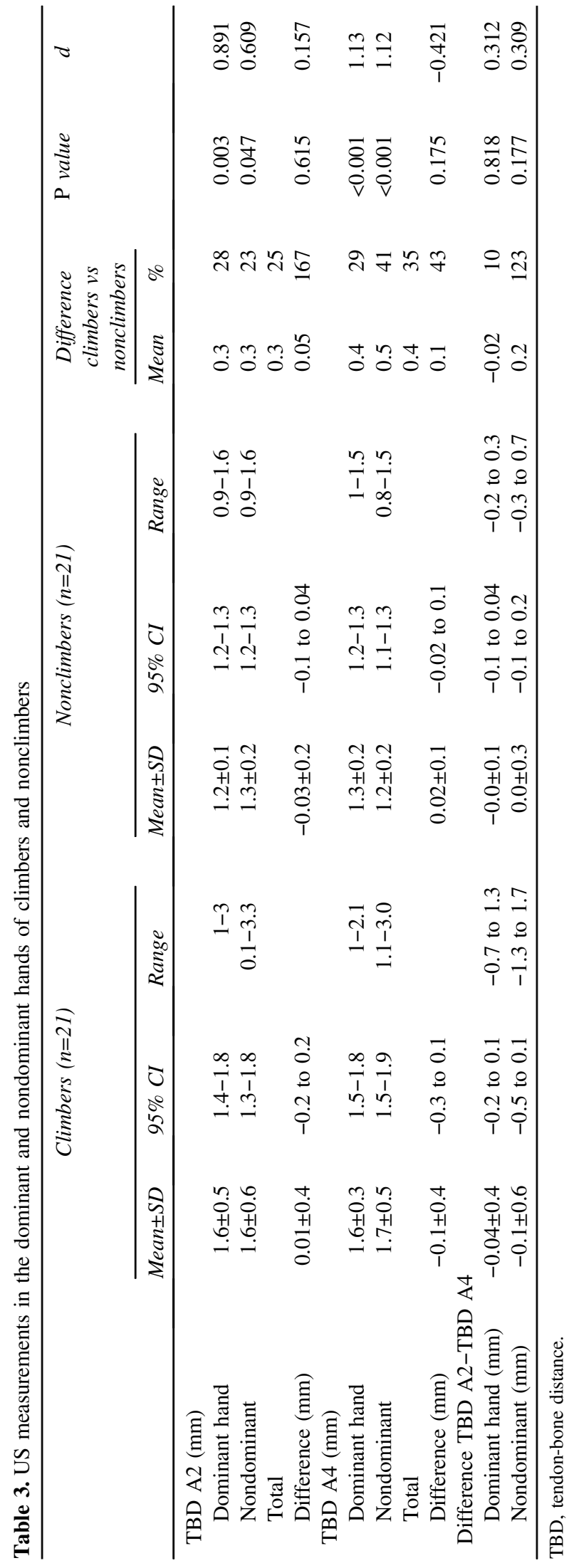

among quantitative variables were assessed through Pearson's coefficient. The confidence level was set at 95\% and significance at $P<0.05$.

\section{Results}

Of the 31 initial climbers ( 3 female and 28 male), 4 climbers ( 3 female and 1 male) did not meet the inclusion criteria of climbing for 15 or more consecutive years above a 9.66 level of difficulty. Although female participants were not excluded a priori, only male climbers met the entry criteria. Consequently, the nonclimbing group consisted of male participants. Of the remaining 27 climbers, 4 were excluded: 3 were diagnosed with complete A2 rupture and 1 with A2, A3, and A4 multiple rupture. Demographic data, performance level, years of climbing experience, and consecutive years of climbing at a level higher than UIAA grade 9.66 are provided in Table 1.

A total of 84 pulleys were measured in each group, half A2 and half A4. In the climbers group, 17 pulleys (20\%; 8 A2 [1.5 $\pm 0.5 \mathrm{~mm} ; 0.9-3.3 \mathrm{~mm}]$ and 9 A4 [1.6 $\pm 0.4 \mathrm{~mm} ; 1-3 \mathrm{~mm}]$ ) were over the $2 \mathrm{~mm}$ TBD threshold, whereas in the nonclimbers group, all pulleys were below the limit value (Table 2). US TBD measurements of A2 and A4 pulleys can be found in Table 3. In the repeated-measures analysis of variance including US measurements for both pulleys, significant interactions were detected for both hands and both groups $(P<0.001)$.

Data recorded for the A2 pulley indicated an overall $25 \%$ greater TBD in climbers compared to nonclimbers. The difference in TBD was $28 \%$ greater for the dominant hand $(P=0.003)$, and the effect size was large. Similarly, TBD in the nondominant hand was $23 \%$ greater $(P=0.047)$, and the effect size was intermediate. No significant differences in A2 pulley TBD were detected between the dominant and nondominant hand $(P=0.615)$.

Our data for the A4 pulley also indicated an overall $35 \%$ greater TBD in climbers compared to nonclimbers. The difference in TBD was $29 \%$ greater for the dominant hand $(P<0.001)$, and the effect size was large. Similarly, TBD in the nondominant hand was $41 \%$ greater $(P<0.001)$, and the effect size was also large. No significant differences in A4 pulley TBD were detected between the dominant and nondominant hand $(P=0.175)$.

Within the group of experienced climbers, TBDs were similar for A2 and A4, although somewhat greater for A4. TBD differences between A2 and A4 were not significant when comparing the groups of climbers with controls. 


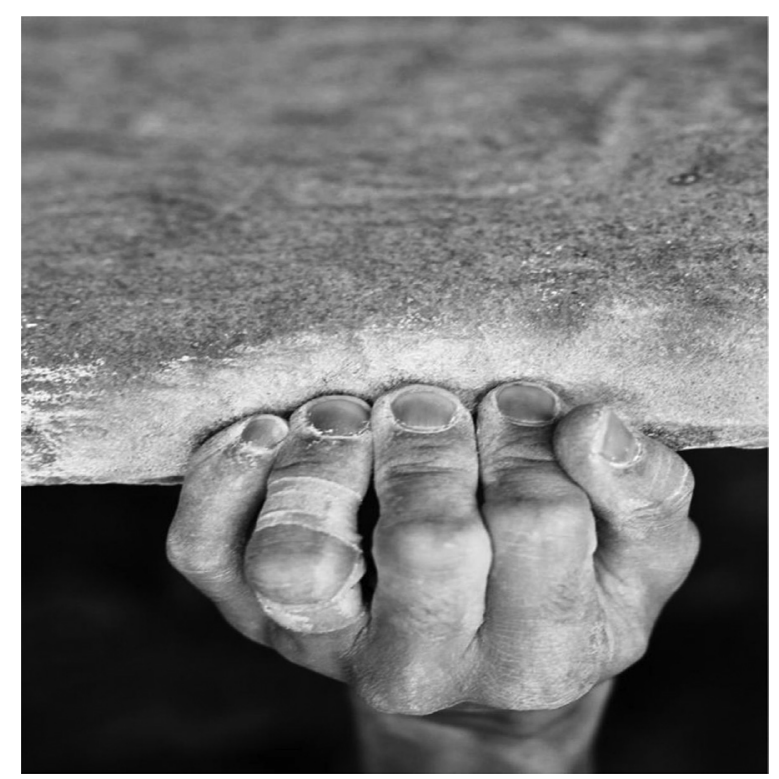

Figure 2. Close crimp grip.

\section{Discussion}

One of the main roles of the musculoskeletal system is to generate different force vectors and thus achieve functional movements. When the capacity to perform these functional movements is exceeded by a determinate workload owing to chronic repetitive trauma, body tissues may react in 2 ways: claudication and injury, or adaptation. ${ }^{22}$ During adaptation, tissue modifications enable the musculoskeletal system to satisfy much more efficiently the biomechanical demands to which the tissue is subjected. ${ }^{23}$ Using different imaging techniques, tissue modifications resulting from a process of adaptation or from a mild injury cannot always be distinguished. Thus, discerning between them depends mainly on the presence or absence of clinical symptoms such as pain or functional limitations. ${ }^{24}$

During the different hand grips employed when climbing, bowstringing of the flexor tendons in the fingers submit the annular pulleys to friction, compression, and traction forces. ${ }^{25}$ The grip that causes the greatest load on the pulleys is the crimp grip (Figure 2), involving at least a $90^{\circ}$ of proximal interphalangeal joint flexion and distal interphalangeal joint hyperextension. ${ }^{26}$ This grip, normally used to grasp small finger holds, involves a load 36 times greater for the A2 pulley compared to the slope grip. ${ }^{27}$ Furthermore, the force incurred by the distal part of the A2 pulley is threefold that supported by the fingertip. ${ }^{28}$ Generally speaking, climbers support a load of $380 \mathrm{~N}$ per finger, yet a 70-kg climber in a single-finger grip may support as much as $450 \mathrm{~N}$, exceeding the reported maximum tolerated load of 380 to $400 \mathrm{~N}$ for the A2 pulley. ${ }^{1}$ Thus, to support this load, a prior period of adaptation is needed. ${ }^{1}$ Accordingly, we would expect anatomic and structural modifications in the fingers allowing climbers to adapt to these biomechanical demands. This is consistent with reported adaptive morphologic and radiologic modifications in bone, A2 and A4 flexor tendon pulleys, and volar plates of the interphalangeal joints in high-level climbers without injury symptoms. ${ }^{7,12}$

\section{US TBD THRESHOLD}

To diagnose a complete A 2 or A4 pulley system rupture, a US TBD of $2 \mathrm{~mm}$ has been described in the literature as the best cutoff. ${ }^{29}$ However, it should be noted that false positives have been detected among asymptomatic nonclimbers when using this threshold. ${ }^{30}$ Although in the present study, this cutoff value was not reached or exceeded in any of the pulleys for the control group, this did occur in $19 \%(1.5 \pm 0.5 \mathrm{~mm}, 0.9-3.3 \mathrm{~mm})$ of A2 pulleys and $21 \%(1.6 \pm 0.4 \mathrm{~mm}, 1-3 \mathrm{~mm})$ of A4 pulleys (20\% of total pulleys) in the asymptomatic experienced high-level climbers group. Thus, the latter could be considered complete pulley ruptures. ${ }^{19}$ A different TBD threshold value that has been proposed, also using active forced flexion finger position, is $5 \mathrm{~mm} .{ }^{20}$ This value was not reached in any of the measured pulleys. All in all, not always will a climber with a TBD considered diagnostic be symptomatic, although the sum of both factors (pain and elevated TBD) along with a reduction in finger grip strength or other accompanying US signs will suggest the diagnosis of a ruptured pulley. ${ }^{17}$

\section{EXPERIENCED HIGH-LEVEL CLIMBERS TBD VS NONCLIMBERS TBD}

Our findings reveal a longer TBD in the A2 and A4 finger pulleys of experienced climbers without symptoms of injury compared to matched healthy nonclimbers. It is certainly feasible that the $25 \%$ and $35 \%$ longer TBD for A2 and A4 pulleys, respectively, could be part of an adaptive mechanism related to long periods of climbing, as for other adaptive modifications that have been described in bone and soft tissues in this population. ${ }^{7,12}$ Another issue worth considering is the possibility of histoarchitectural failure in the retention capacity of the pulley system, or even the formation of small subclinical ruptures over time, as occurs in chronic tendinopathies involving degeneration and intrasubstance tears of the tendon. ${ }^{31}$ Future studies should determine whether this increase in TBD occurs progressively as an adaptation process or occurs suddenly after an acute period of 
overloading the fingers due to an increased workload, the latter being more suggestive of underdiagnosed pulley tear.

Bowstringing and thickening as physiological adaptations or subclinical entities of A2 and A4 pulleys show similar trends. Pulley thickness was found to be significantly higher in climbers vs nonclimbers, ${ }^{7}$ and in the present study the same trend was found for TBD. A4 showed greater changes when submitted to longstanding overloading, with $75 \%$ thickening and $35 \%$ bowstringing in comparison to A2 (69\% and 25\%, respectively). Thus, the annular pulleys show a significant tendency to both thicken and slacken. ${ }^{7}$ Overall, what seems important about a synchronic thickening and slackening process is that, if a pulley thickens without gaining laxity, the space between the flexor and pulley would be reduced, increasing friction and giving rise to the condition of trigger finger.

\section{A2 TBD VS A4 TBD}

We report an overall $10 \%$ higher TBD in A4 pulleys compared to the A2 pulleys analyzed, although the difference is not statistically significant. One hypothesis to explain this is the difference in the breaking load between pulleys. The mean perpendicular forces acting on $\mathrm{A} 2$ and A4 pulleys were approximately equal $(254.8 \mathrm{~N}$ and $220.9 \mathrm{~N}$ ) when performing a crimp grip. ${ }^{27}$ The maximum breaking load is $407.5 \mathrm{~N}$ for the A2 pulley and $209.5 \mathrm{~N}$ for the A4 pulley, which means that A2 is twice as strong as $\mathrm{A} 4 .{ }^{32}$ In a maximum crimp grip load, A2 can easily accommodate such forces, whereas A4 is at the limit of its load accommodation capacity. According to this rationale, it is easier for a climber to rupture an A4 pulley than A2 pulley. ${ }^{33}$ However, over a minimum of 15 y sustaining high-load repetitive crimp grips and without any pulley rupture, greater asymptomatic bowstringing could be more likely in the A4 pulley than in the A2 pulley.

No difference in TBD was noted here between the dominant and nondominant hand in both climbers and nonclimbers. This was not unexpected because climbing is a relatively symmetric sport.

\section{LIMITATIONS}

The main limitations of this study were its small sample size, the lack of female participants, and the absence of intraobserver or interobserver variability. A drawback was that no previous medical records were reviewed when applying the exclusion criteria regarding previous diagnosis of pulley rupture of the ring finger or hand surgery; this information was obtained by interviewing the sample participants, and this might have incurred a self-reporting bias. Therefore, a new study should be developed with the purpose of determining the factors that may modify TBD.

\section{Conclusions}

Asymptomatic experienced high-level climbers have significantly longer A2 and A4 flexor pulley TBDs (25\% and $35 \%$, respectively) compared to asymptomatic nonclimbers. Such differences suggest a subjacent adaptive process to elevated workloads over the years or the possibility of underdiagnosed annular pulley ruptures. The present results should be taken into consideration by sonographers when performing A2 or A4 pulley US assessments in experienced high-level climbers to avoid false positives. The $2 \mathrm{~mm}$ TBD threshold to diagnose a complete A2 or A4 pulley rupture could be excessively sensitive for such climbers.

Author Contributions: Study concept and design (XI, MB); acquisition of the data (XI, AD, LG); analysis of the data (ESR); drafting of final manuscript (XI, JDLF, FD, MB); critical revision of the manuscript (XI, JDLF, ESR, FD, MB); approval of final manuscript (all authors).

Financial/Material Support: None.

Disclosures: None.

\section{References}

1. King EA, Lien JR. Flexor tendon pulley injuries in rock climbers. Hand Clin. 2017:33(1):141-8.

2. Schöffl VR, Hoffmann G, Küpper T. Acute injury risk and severity in indoor climbing - a prospective analysis of 515, 337 indoor climbing wall visits in 5 years. Wilderness Environ Med. 2013;24(3):187-94.

3. Woollings KY, McKay CD, Emery CA. Risk factors for injury in sport climbing and bouldering: a systematic review of the literature. Br J Sports Med. 2015;49(17):1094-9.

4. Schweizer A. Sport climbing from a medical point of view. Swiss Med Wkly. 2012;142:w13688.

5. Zafonte B, Rendulic D, Szabo RM. Flexor pulley system: anatomy, injury, and management. J Hand Surg Am. 2014;39(12):2525-32.

6. Lutter C, Hotfiel T, Tischer T, Lenz R, Schöffl V. Evaluation of rock climbing related injuries in older athletes. Wilderness Environ Med. 2019;30(4):362-8.

7. Schreiber T, Allenspach P, Seifert B, Schweizer A. Connective tissue adaptations in the fingers of performance sport climbers. Eur J Sport Sci. 2015;15(8):696-702.

8. Schöffl V, Morrison A, Hefti U, Ullrich S, Küpper T. The UIAA medical commission injury classification for mountaineering and climbing sports. Wilderness Environ Med. 2011;22(1):46-51.

9. Schöffl VR, Hochholzer T, Imhoff AB, Schöffl I. Radiographic adaptations to the stress of high-level rock climbing in junior athletes. Am J Sports Med. 2007;35(1):86-92. 
10. Bayer T, Schöffl VR, Lenhart M, Herold T. Epiphyseal stress fractures of finger phalanges in adolescent climbing athletes: a 3.0-tesla magnetic resonance imaging evaluation. Skeletal Radiol. 2013;42(11):1521-5.

11. Schöffl V, Popp D, Küpper T, Schöffl I. Injury trends in rock climbers: evaluation of a case series of 911 injuries between 2009 and 2012. Wilderness Environ Med. 2015;26(1):62-7.

12. Meyers RN, Hobbs SL, Howell DR, Provance AJ. Are adolescent climbers aware of the most common youth climbing injury and safe training practices? Int J Environ Res Public Health. 2020;17(3):812.

13. Hahn F, Erschbaumer M, Allenspach P, Rufibach K, Schweizer A. Physiological bone responses in the fingers after more than 10 years of high-level sport climbing: analysis of cortical parameters. Wilderness Environ Med. 2012;23(1):31-6.

14. Garcia K, Jaramillo D, Rubesova E. Ultrasound evaluation of stress injuries and physiological adaptations in the fingers of adolescent competitive rock climbers. Pediatr Radiol. 2018;48(3):366-73.

15. Lapegue F, Andre A, Brun C, Bakouche S, Chiavassa H, Sans N, et al. Traumatic flexor tendon injuries. Diagn Interv Imaging. 2015;96(12):1279-92.

16. Schöffl I, Hugel A, Schoffl V, Rascher W, Jüngert J. Diagnosis of complex pulley ruptures using ultrasound in cadaver models. Ultrasound Med Biol. 2017;43(3):662-9.

17. Iruretagoiena-Urbieta $X$, De la Fuente-Ortiz de Zarate J, Blasi M, Obradó-Carriedo F, Ormazabal-Aristegi A, Rodríguez-López ES. Grip force measurement as a complement to high-resolution ultrasound in the diagnosis and follow-up of the A2 and A4 finger pulley injuries. Diagnostics (Basel). 2020;10(4):206.

18. World Medical Association Declaration of Helsinki: ethical principles for medical research involving human subjects. J Am Coll Dent. 2014;81(3):14-8.

19. Iruretagoiena-Urbieta $X$, De la Fuente-Ortiz de Zarate J, Rodríguez-López ES, Barceló-Galíndez P, Oliva-PascualVaca A, Otero-Campos A, et al. Ultrasonographic diagnosis of A2 or A4 flexor tendon pulley injury: a systematic review. Wilderness Environ Med. 2020;31(4):498-505.

20. Bodner G, Rudish A, Gabl M, Judmaier W, Springer P, Klauser A. Diagnosis of digital flexor tendon annular pulley disruption: comparison of high frequency ultrasound and MRI. Ultraschall Med. 1999;20(4):131-6.
21. Schöffl VR, Einwag F, Strecker W, Schöffl I. Strength measurement and clinical outcome after pulley ruptures in climbers. Med Sci Sports Exerc. 2006;38(4):637-43.

22. Chang CY, Torriani M, Huang AJ. Rock climbing injuries: acute and chronic repetitive trauma. Curr Probl Diagn Radiol. 2016;45(3):205-14.

23. Bohm S, Mersmann F, Arampatzis A. Human tendon adaptation in response to mechanical loading: a systematic review and meta-analysis of exercise intervention studies on healthy adults. Sports Med Open. 2015;1(1):7.

24. McAuliffe S, McCreesh K, Culloty F, Purtill H, O’Sullivan K. Can ultrasound imaging predict the development of Achilles and patellar tendinopathy? A systematic review and metaanalysis. Br J Sports Med. 2016;50(24):1516-23.

25. Moor BK, Nagy L, Snedeker JG, Schweizer A. Friction between finger flexor tendons and the pulley system in the crimp grip position. Clin Biomech (Bristol, Avon). 2009;24(1):20-5.

26. Schöffl I, Oppelt K, Jüngert J, Schweizer A, Neuhuber W, Schöffl V. The influence of the crimp and slope grip position on the finger pulley system. $J$ Biomech. 2009;42(13):2183-7.

27. Vigouroux L, Quaine F, Labarre-Vila A, Moutet F. Estimation of finger muscle tendon tensions and pulley forces during specific sport-climbing grip techniques. $J$ Biomech. 2006;39(14):2583-92.

28. Schweizer A. Biomechanical properties of the crimp grip position in rock climbers. J Biomech. 2001;34(2):217-23.

29. Schöffl V, Hochholzer T, Winkelmann HP, Strecker W. Pulley injuries in rock climbers. Wilderness Environ Med. 2003;14(2):94-100.

30. Bassemir D, Unglaub F, Hahn P, Müller LP, Bruckner T, Spies CK. Sonographical parameters of the finger pulley system in healthy adults. Arch Orthop Trauma Surg. 2015;135(11):1615-22.

31. Cook JL, Rio E, Purdam CR, Docking SI. Revisiting the continuum model of tendon pathology: what is its merit in clinical practice and research? $\mathrm{Br} J$ Sports Med. 2016;50(19):1187-91.

32. Lin GT, Cooney WP, Amadio PC, An KN. Mechanical property of human pulleys. J Hand Surg Br. 1990;15(4):429-34.

33. Marco RA, Sharkey NA, Smith TS, Zissimos AG. Pathomecanics of closed rupture of the flexor tendon pulleys in rock climbers. J Bone Joint Surg Am. 1998;80(7):1012-9. 